\title{
EXAMPLES OF MEDICAL IMAGING AND MEASUREMENTS BASED ON THE NONINVASIVE TISSUE TRANSILLUMINATION
}

\author{
A. CYSEWSKA-SOBUSIAK, A. HULEWICZ, Z. KRAWIECKI \& G. WICZYNSKI \\ Institute of Electrical Engineering and Electronics, Poznan University of Technology, Poland.
}

\begin{abstract}
The paper is devoted to modern diagnostic methods: based on utilization of the detectable effects of light-tissue interaction. Transillumination is a method of object examination by the passage of light through tissues or a body cavity. Under the transillumination and illumination from underneath, it is possible to diagnose and monitor the parameters of tissues and organs examined. The authors briefly report the current state in the art as well as present their own results. Among other things, the presented examples include: optoelectronic techniques used in monitoring of the living tissues vitality and promising results obtained during preliminary experiments with transillumination scanning applied to human hand fingers. Upon the test results analysis, the necessity arises to configure the optical part so that a better resolution can be obtained with capacity of effective transillumination of objects optically thicker.
\end{abstract}

Keywords: Light-tissue interaction, medical imaging, noninvasive biomeasurements, optoelectronics, transillumination.

\section{INTRODUCTION}

The role of optical techniques in current medical measurements and imaging is especially significant [1-5]. Advances in medical and biological technology are due to the increasing interaction and collaboration between medical and engineering scientists. There is also necessity to work well in a well-integrated interdisciplinary team. Efficient and safe application of light-tissue interactions to biomedical engineering must meet the various human and technical needs, such as medical practice, ethics, clinical care of patients, instrumentation reliability, and material biocompatibility. Different imaging methods allow detecting different properties of tissues through a variety of utilized phenomena. The interest in development of optical techniques in biomedicine to obtain images of tissues and organs is great and still growing. The different parts of the electromagnetic spectrum have very different effects upon interaction with biological objects. Red light and near-infrared radiation emitted by high-efficient LEDs can be noninvasively transmitted through the blood-supplied tissues. The-stateof-the-art, taken here into account as the background, refers to the reported optical parameters of tissues when exposed to wavelengths of light included in the optical window in tissue spectrum $[1,6]$.

When a biological object is exposed to illumination, we can receive the selective optical response to particular wavelengths. Intensity of light and other factors affecting human tissues has to be sufficiently low. Temperature exceeding $41^{\circ} \mathrm{C}$ can cause tissue damage.

An optoelectronic sensor based either on reflection or transmission variant of light-tissue interaction can be used to detect the photoplethysmography (PPG) signal; however, there are a lot of difficult measurement problems. The examples presented by the authors include promising results obtained during experiments with transillumination scanning applied to human hand fingers. Previous own authors' experience in one-dimensional modeling for transmission pulse oximetry and PPG $[7,8]$ has been adapted. The presented work is an extended and revised version of the paper presented at the First International Conference on Light in Engineering, Architecture and the Environment held in May in Poland [9]. 


\section{CHARACTERISTICS OF OPTICAL PROPERTIES OF HUMAN TISSUES}

The interactions occurring between the light and the human tissues result in scattering, absorption and fluorescence, providing information on the structure, physiology, biochemistry, and molecular functions. Optical imaging is used for description of surface and volume structures.

Tissues are optically turbid media that are highly scattering. A typical scattering coefficient for visible light in human tissues is $100 \mathrm{~cm}^{-1}$ in comparison with $0.2 \mathrm{~cm}^{-1}$ for X-rays used in medical diagnostics.

Among the applied methods of tissue parameters measurement, a tendency to develop methods based on detection and analysis of natural and forced bio-optical phenomena is significant. Biosignals can be acquired in a variety of ways. Bio-optical signals are generated by the optical attributes of biological systems. Measurable effects of interaction between light and tissues may be used in biomedical engineering with emission, reflection or transmission mode, respectively. There is a specific window extending from 600 to $1200 \mathrm{~nm}$ in which optical radiation can penetrate into human tissue set and propagate through it during transillumination (Fig. 1). However, due to strong scattering of the light, the practical implementation of optical transillumination for medical imaging is a difficult task. Measurements are very rich in artifacts, noises and disturbances that affect processing and can cause the decrease in useful output signals.

Reports in the literature often give the particular information about parameters, for example, percentage transmission or reflection of light measured at the particular wavelength penetrating a particular human or animal organ $[1,2,6]$. Many reports refer to data collected in vitro or in vivo, but always invasively. Inhomogeneities in the living complex structures must lead to a spread in measured values when compared to those obtained for the selected homogeneous samples. A particular tissue composition very depends on blood and water contents what results in differences between values of optical parameters if to determine them at several compositions of various kinds and size. There are several sources of optical inhomogeneity of a biological object, which can be described mainly as structural imperfections and optical anisotropy. Depending on the locality and diameter of the light beam passing through the object, the effects of light-tissue interaction can differ very much. The specific human blood feature is that hemoglobin which is carrying oxygen (oxyhemoglobin $\mathrm{HbO}_{2}$ ) absorbs light in the IR region of the spectrum; hemoglobin that isn't carrying oxygen (reduced hemoglobin $\mathrm{Hb}$ ) absorbs visible red light [2].

\section{ADVANTAGES OF TRANSILLUMINATION}

Tissue transillumination means the passing of a strong beam of light through a tissue sample or a part of the body for medical inspection, at the wavelengths included in the window shown in Fig. 1 . Through forced transillumination in direction assumed, a bio-optical signal may be obtained

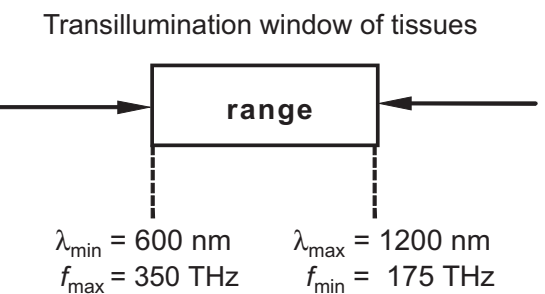

Figure 1: Transillumination window for human tissues. 
for diagnostic purposes. The transilluminated object examined is the optical link element where it behaves as the radiation intensity modulator.

By definition, an optoelectronic sensor is a device that produces an electrical signal proportional to the amount of light incident on its active area [3,4]. These sensors still become more efficient, portable, and smaller. The methods used in positioning objects in relation to the sources and detectors of optical radiation are presented in Fig. 2.

The intensity transmission expresses the efficient relative measure of beam propagation effectiveness and the effectiveness of bio-optical signal detection and its transformation into photoelectric current largely depends on the metrological properties of a source of the incident light and detector of the light transmitted by an object.

The sensor types include: light-to-voltage converters, light-to-frequency converters, ambient light sensors, linear sensor arrays, color sensors, and reflective light sensors [3]. Currently, LEDs play an important role in many applications and will play a much larger role in the future illumination and other areas [11,12]. White, red, and near infrared LEDs have potential to be also used in in vivo medical measurements and imaging taking into account specific selective properties of light absorption and scattering by human tissues. Optoelectronic sensors usually combine a set of LEDs emitting the incident light (600 to $1000 \mathrm{~nm}$ ) as the photoemitter with a broadband photodiodes (350 to $1100 \mathrm{~nm}$ ) as the photodetector of transmitted or reflected light. Modern high-efficient LEDs are very useful as moderately narrowband photoemitters with an approximately Gaussian spectral shape. The silicon PIN photodiodes may be used in practice as photodetectors. These small and lightweight elements are very sensitive, have low noise levels, and convert the light into electronic signal linearly, in the whole measuring window and over a large dynamic range.

A current driving LEDs intensity makes the sensor to be 'smart' by means to control levels of the transmitted light and provide incident light, which might be transmitted through various real objects, as being more or less thick and pigmented. Evidently, a way the photoemitter is driving by current impulses as well as the selective photocurrent is converted, amplified and conditioned are also critical [4,5].

As mentioned, transillumination is the phenomenon of transmitting optical radiation with defined parameters by a given object, which becomes the carrier of information about the characteristics of this object.

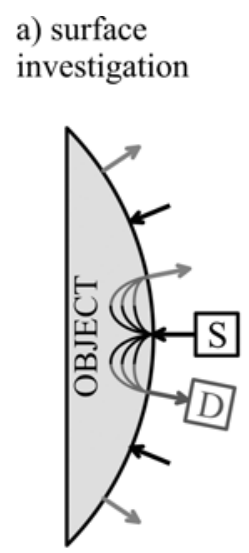

\begin{abstract}
b) investigation through object
\end{abstract}

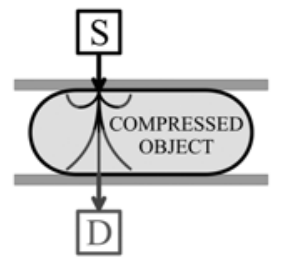

c) spatial investigation

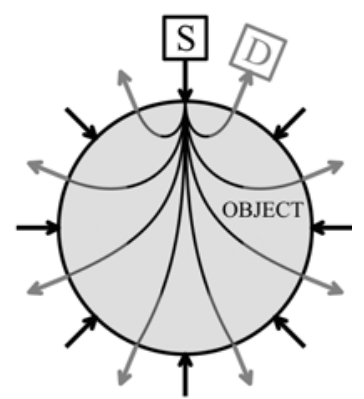

Figure 2: Examples of three variants of the investigated object position in relation to optoelectronic sensors containing the photo-emitters (light sources S) and photo-detectors, D [10]. 
Continuous monitoring of light transmitted in the penetrated region could offer a control about the changes inside the objects and become important in predicting the light action outcome. Representative examples of transillumination may include:

- detection of body fluids components (spectrophotometry, oximetry in vivo),

- localization of veins, cysts, and neoplasms from underneath,

- transillumination with white light (instead of X-rays),

- imaging and monitoring of pulse wave (PPG),

- transmission variant of pulse oximetry, and

- optical tomography.

Optical properties of transilluminated or illuminated tissues or organs depends on strong light absorption and scattering - numerous issues related to the result interpretation still remain unsolved. A given set of living tissues consists of many components that create the determined spatial configuration. Light transmitted through tissues is classified into three categories: ballistic light, quasi-ballistic light, and diffuse light. Depending on the locality and diameter of the light beam passing through the object, the effects of light-tissue interaction can differ very much. Optical radiation that is to play the role of an effective information carrier should be sufficiently coherent and, due to the high optical density of the object, should also have possibly high intensity. However, for higher power density quantities, some destructive photothermal effects can occur. Wavelength and power of radiation selected improperly may not only act ineffectively but also cause damage or destruction of the object.

\section{MONITORING OF ARTERIAL PULSE WAVE AND OXYGEN SATURATION}

Over the last years, the development of new and different medical equipment is impressive. Biomedical signals carry information that is often enclosed in a signal shape. The most common example of transillumination is the observation of arterial blood pulsation allowing for measuring the blood pulse and blood oxygenation $[2,13]$. The PPG signal, which reveals circulatory depression and arrhythmia, may be acquired as a waveform to which the pulse wave contributes as the main component [1,2]. Measurements of arterial blood gases and $\mathrm{pH}$ provide information about the adequacy of blood oxygenation and $\mathrm{CO}_{2}$ elimination. Knowing what percentage of the hemoglobin is saturated with oxygen is important when administering anesthesia as well as for helping to diagnose various diseases.

All traditional in vitro measurements of blood oxygen content need to draw blood samples are risky, rich in complications and very time-consuming. Early evaluations of blood oxygenation with noninvasive, transcutaneous techniques based on light-tissue interaction were unacceptable in practice; however, the progress which has been made in this area is excellent. Credit for the great present interest in these measurements belongs to anesthesiologist William New who with engineer Jack Lloyd founded, in USA Nellcor Incorporated, [14] the first commercial device called pulse oximeter in 1985. Today, various models are manufactured and used in a lot of countries in the world. However, till date, the method employed is a subject of continuous development and improvement.

\subsection{PPG and pulse oximetry}

The modulations of bio-optical signals, induced in tissues by the arterial blood pulsations, are the basis of very important optical bloodless diagnostic methods: PPG and pulse oximetry $[2,8]$ make it possible to monitor the living tissues vitality noninvasively. 
The PPG signal is acquired as a result of noninvasive transillumination of well blood-supplied layer of tissues [1-3]. The main component of each PPG signal is a pulse wave which waveform reflects the hemodynamic phenomena (i.e. changes of arterial blood volume) occurring synchronously with heart rate in blood vessels. Another significant component of PPG signal is the slowly varying component, the sources of which are:

- Slight respiratory movements of the patient monitored,

- Motion artifacts caused by significant relocations of the patient or change of the object monitored orientation in relation to the immobile reference system.

The biomedical signals including but not limited to the PPG signal are 'difficult' both due to the specific requirements related to the acquisition methods and the presence of numerous artifacts significantly impeding further digital conditioning [2-6].

The analysis of PPG with the simultaneous monitoring of ECG makes it possible to decrease the influence of artifacts and produces accurate results in patients with often poor peripheral circulation. Pulse oximetry, which is currently known as one of the most significant advance made in patient monitoring, is based on noninvasive use of two phenomena: natural arterial pulsations and differences in optical properties of blood and other tissues. This technique smartly joins rules of both in vivo spectrophotometry and PPG to monitor the arterial blood oxygen saturation $\mathrm{SaO}_{2}=\mathrm{HbO}_{2}$ / $\left(\mathrm{Hb}+\mathrm{HbO}_{2}\right)$. What is unique in pulse oximetry is that it is possible to sense the global oxygen saturation of human body arterial blood by noninvasive transillumination of only a peripheral tissue set which allows us to see a 'representative' arterial blood in other tissue components. The principle is simple and is based on spectrophotometry rules, referring to idealized blood consisting of only two absorbers to be detected.

The transmission variants of light-tissue interaction that is accomplished with the object transillumination can be often more convenient and sensitive than the reflection variants (Fig.3). In practice, the measurements are accomplished with optoelectronic sensors placed on 'living cuvettes' such as, for example, finger-tips, earlobes, nasal bridges etc. The finger tips are the especially useful sites to place the sensors which are applied directly and very often in prolonged duration.

The thickness of the object varies with each pulse, changing the light path length which effects are eliminated when estimating the oxygen saturation. The difference in shape and stability of the peripheral PPG waveform can be used as an indication of possible motion artifacts or low perfusion conditions (Fig. 4) [15]. The analysis of the phenomena related to the pulse wave propagation and changes of its curve shape taking place in within the vascular system can require the simultaneous

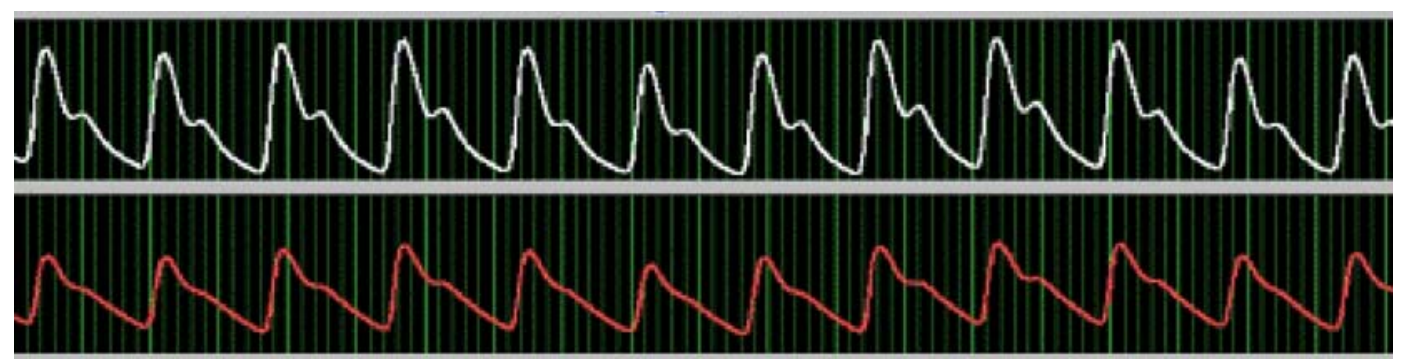

Figure 3: Examples of PPG signals obtained with a reflection finger sensor (top) and a transmission finger sensor (bottom). 


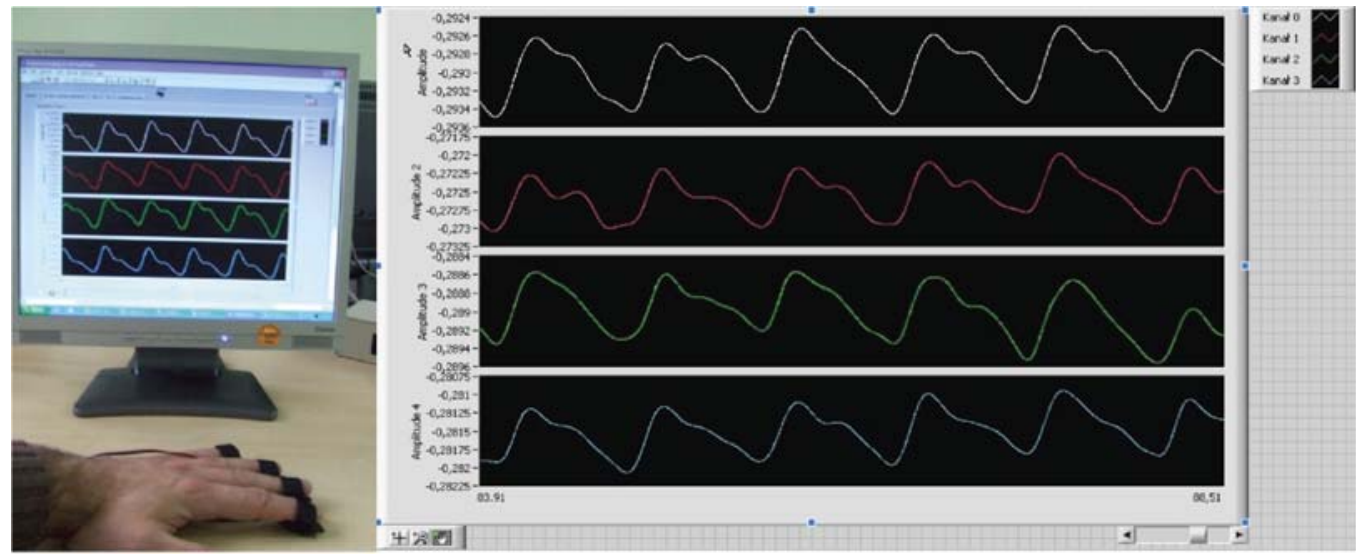

Figure 4: Example of some raw pulse waves acquired from the fingers [15].

detection of the PPG signal in various points of the organism. The data recorded by several sensors may be useful for searching and detecting the irregularities in the operation of the cardiovascular system.

Similarly, if the patient's heart rate displayed by the pulse oximeter differs considerably from the actual heart rate, the displayed saturation value should be questioned.

\subsection{Novel expanded use of the transmission variant of pulse oximetry concept}

A modified approach to the transmission variant of pulse oximetry that was used by the authors in their own studies [16] depends on controlling tendency to changes in the selective components of light transmitted by an examined body site. The components of two raw pulsatile optical signals from the object (Fig. 5), to be obtained at each of both wavelengths (i.e. $U_{\mathrm{RED}}$ and $U_{\mathrm{IR}}$ ), can be processed to four values of voltage which correspond to a reference bottom $\left(U_{\mathrm{REDb}}, U_{\mathrm{IRb}}\right)$, and a peak difference of the arterial pulse wave $\left(U_{\text {REDp }}, U_{\text {IRp }}\right)$ in each heart beat. Output signals from the sensor are held in the desirable range of values by assignment of current pulses which control the photoemitter. The real relationship between a ratio: $\left(U_{\mathrm{REDp}} / U_{\mathrm{REDb}}\right) /\left(U_{\mathrm{IRp}} / U_{\mathrm{IRb}}\right)$ and $\mathrm{SpO}_{2}$, which values estimate $\mathrm{SaO}_{2}$, has been established experimentally. During comparative laboratory studies, the authors of this paper used the modern devices shown in Fig. 6.

A novelty is continuous tracing of the components of optical signals acquired from the transilluminated object. Indicating pulse waveforms and changes in the raw PPG signals at both measuring wavelengths allow the end-user to assess in real time the quality and reliability of the measurements. First, the shape and stability of the PPG curve can be used as an indication of possible influences. Second, by controlling a tendency to changes in raw values of quantities involved in the known nominal processing function, falsely true readings have potential to be detected and corrected.

On the one hand, studying the relationships between either pathological changes in a circulatory rhythm or measurement disturbances and corresponding them to the changes in the frequency spectrum of the photoplethysmogram detected from a peripheral body site may be useful in practice. On the other hand, computer creation and analysis of virtual pulse waveforms can be useful to simulate real signals occurring in pathological situations when measurements are impossible because of either ethical or technical limitations. The obtained results may be useful in computer-aided 


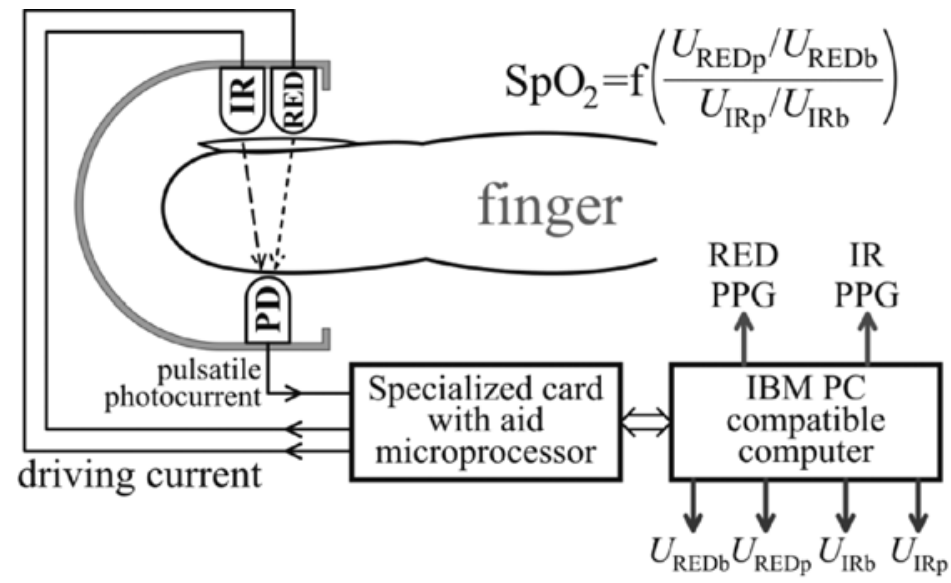

Figure 5: Scheme of the testing device based on a novel use of the pulse oximetry concept.
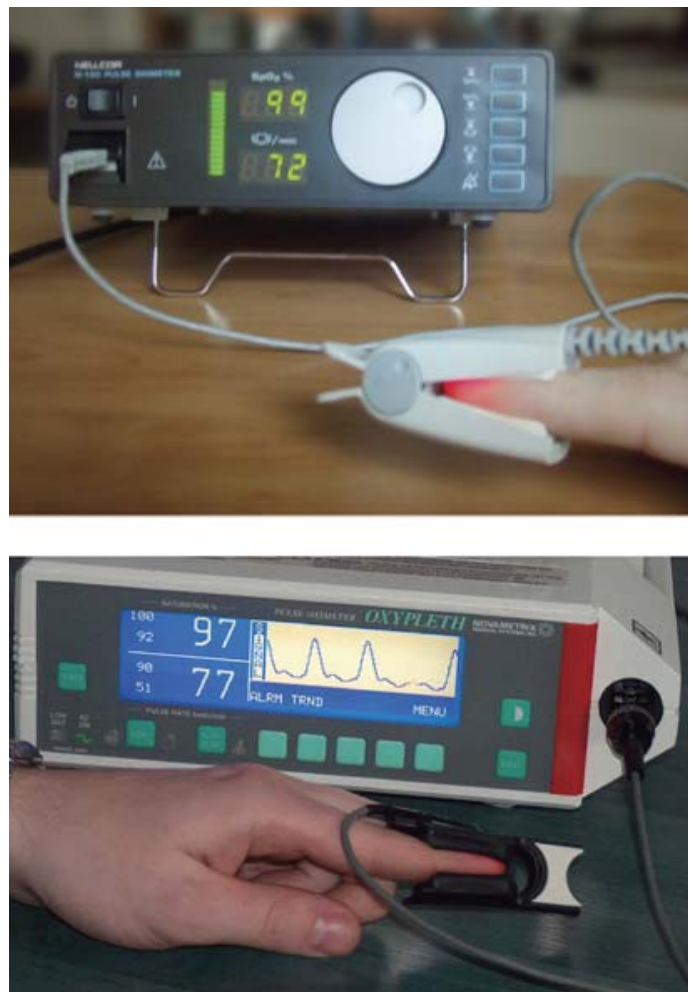

Figure 6: Examples of the measurement results obtained by the authors during their studies with two models of pulse oximeters: Nellcor-180 (top), and Oxypleth Novametrix (bottom). The latter is equipped with a PPG waveform monitoring system. 
generation of reference data for evaluation of light-tissue spatial transillumination. Computer models in particular have been increasingly successful in simulating bio-optical phenomena.

\section{OPTICAL IMAGING OF HAND FINGERS}

In 1895, Wilhelm Conrad Roentgen made the first radiogram of a palm, starting the development of noninvasive image diagnostics methods. Currently, different imaging methods are able to detect different properties of investigated tissues through a variety of phenomena to be utilized. The role of optical techniques in current tendency to develop combined medical imaging is especially significant in such a field as, for example, the modern video endoscopy [5].

Light scattering and absorption can complicate the transillumination image. The optical image resolution can be lower than that of X-ray images; however, it enables to provide information on the functional conditions unavailable in the RTG technique. The system presented in Fig. 7 makes it possible to study the transillumination effects in the optical range, including the visible and near infrared radiation (up to $1000 \mathrm{~nm}$ ). An illuminating source consists of a set of LEDs, emitting the radiation at the wavelength $645 \mathrm{~nm}$ and $880 \mathrm{~nm}$, respectively.

The possibility of efficient transillumination greatly depends on the transmission properties of the object as well as the spectral characteristics of the LEDs and camera. The UI-1240ME monochromatic camera with USB 2.0 interface features a CMOS sensor in 1.3 Megapixel resolution $(1280 \times$ 1024 pixels) [17]. The selected camera without an IR-cut filter makes it possible to register images in the spectral range of 400-1000 nm. Independently from the LED spectrum, the monochromatic camera allows obtaining of imaging in the gray scale.

It is possible to distinguish these structures of the object that occur sufficiently near the camera input surface. Thus, the observation of the internal structures is possible by transillumination of such objects as hands, feet, breasts, etc. Strong light scattering by human tissues makes it hard to observe and evaluate the structures occurring more deeply.

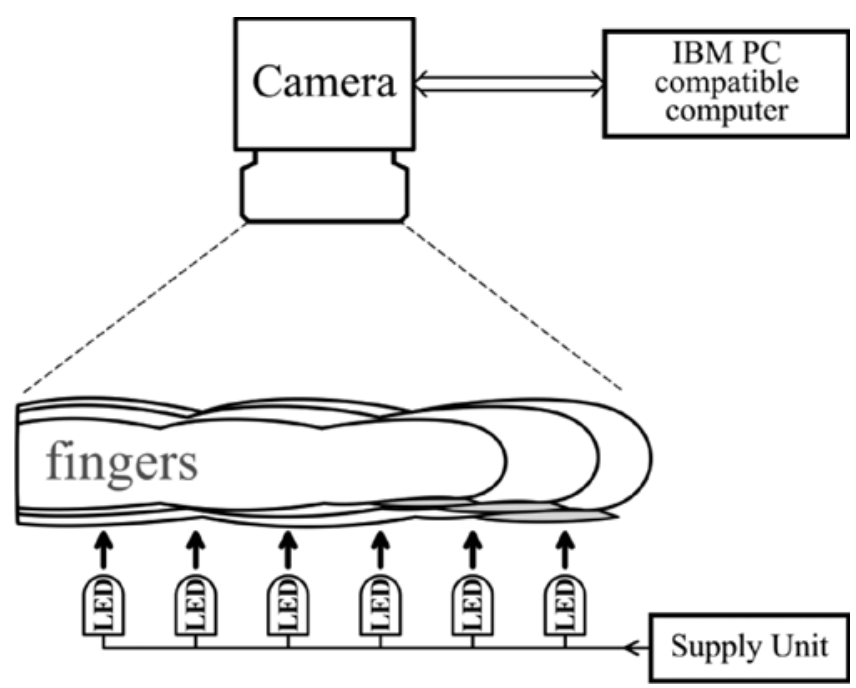

Figure 7: Block diagram of a system designed to transillumination the object; the light source consists of LEDs emitting light at $880 \mathrm{~nm}$ and $645 \mathrm{~nm}$, respectively, and the Camera UI-1240ME-M is the receiver used to detect the light transmitted by the object. 
a)
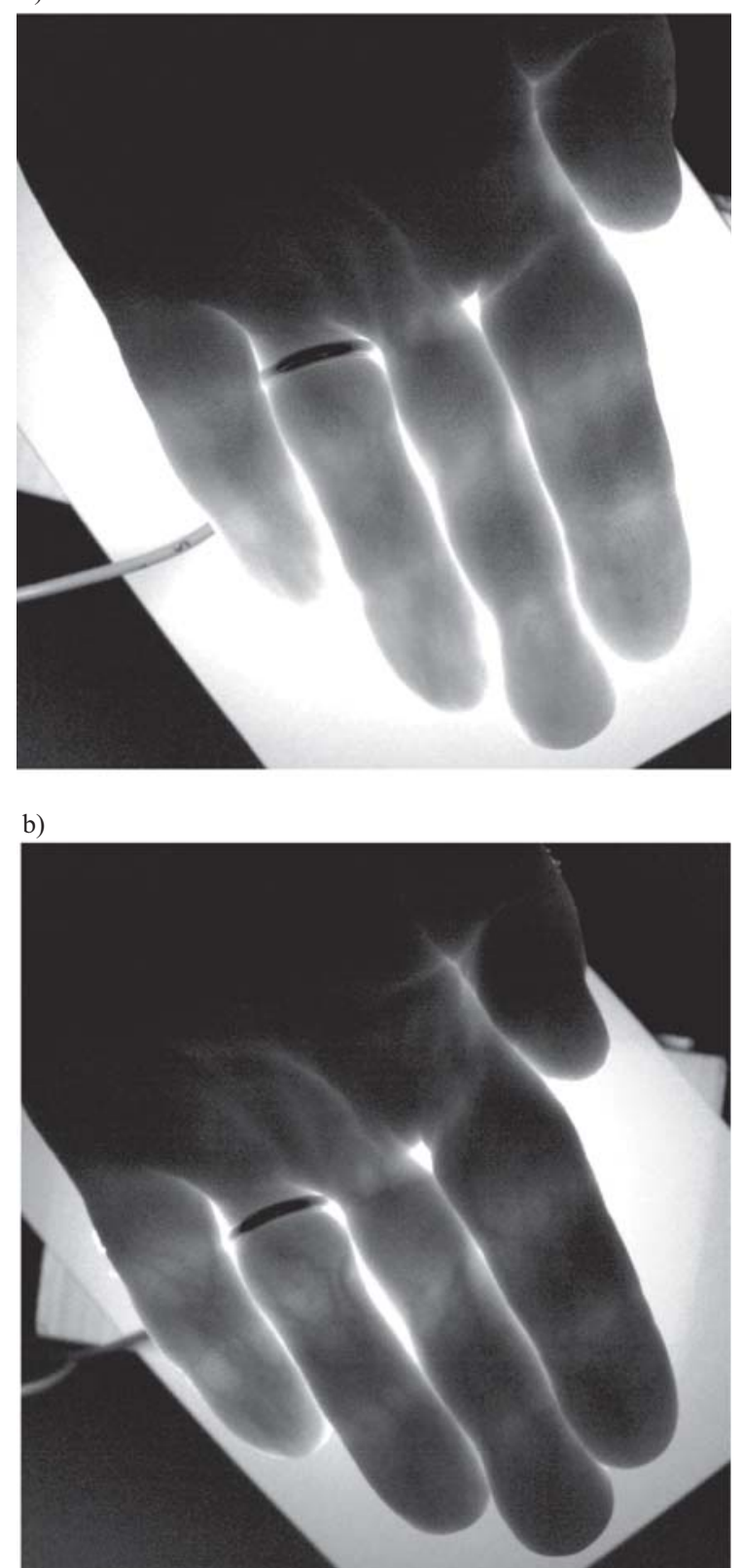

Figure 8: Two images obtained during the hand transillumination at the wavelength $645 \mathrm{~nm}$ (a) and $880 \mathrm{~nm}(\mathrm{~b})$.

Examples of the acquired transillumination images of a hand are presented in Fig. 8. These images have been obtained with the system, block diagram of which is shown in Fig. 7.

The experimental transillumination of hand fingers and foot toes is possible in a quite simple as well as efficient transmitting-receiving system presented in Figs. 9 and 10 [18]. The mechanical 


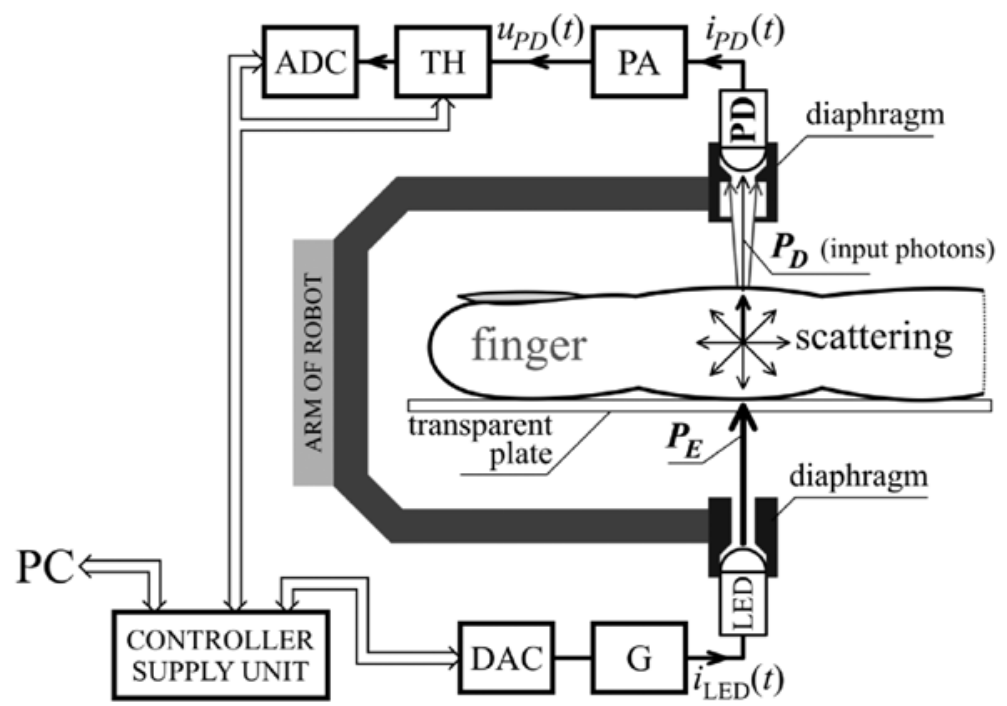

Figure 9: System performed for the impulse transillumination of a finger: PD - photodiode (as photodetector), PA - amplifier and converter of photocurrent $i_{P D}(t), \mathrm{TH}-$ trace-and-hold converter, ADC - analog-to-digital converter, LED - photoemitter, G - source of current pulses $i_{L E D}(t)$, DAC - digital-to-analog converter, $P_{E}$ - radiation illuminating the object, $P_{D}$-radiation detected by photodiode PD.

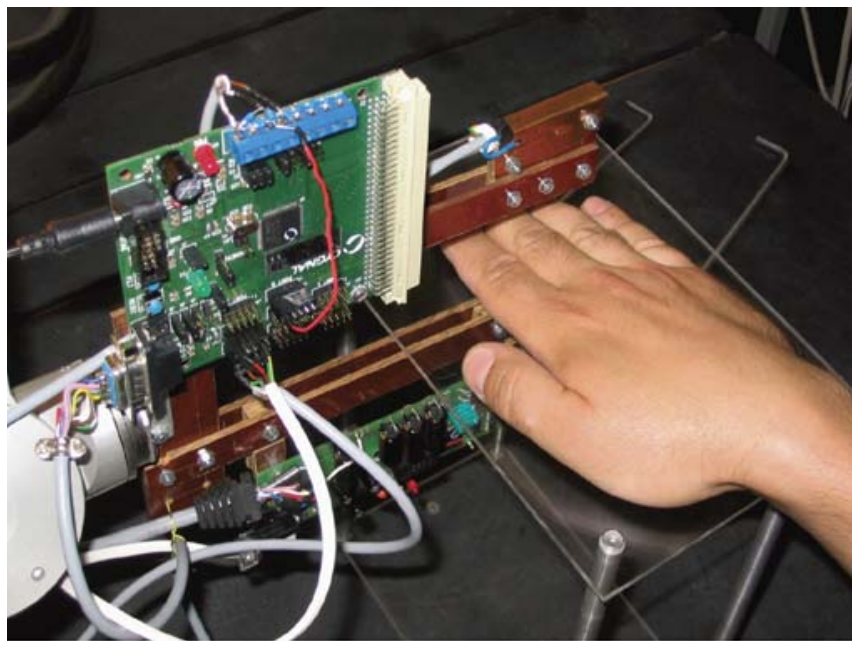

Figure 10: View of a performed transillumination system joined with the robot arm. The input: the transverse motion of the scanning system in relation to the fingers.

structure of this system was constructed in the form of letter C fixed to the robot's arm. A LED diode and photodiode were fit at the structure ends in optical channels of $3 \mathrm{~mm}$ diameter and about $20 \mathrm{~mm}$ length.

The robot arm assembly is flexible and contains the motorization, brakes, motion transmission mechanisms, cable bundle, and pneumatic and electrical circuits for the user. A scanning system has 
been joined with the robot arm. The object's scanning is made in a rectangular $x-y$ coordinates system. The hand examined is laid on a transparent plate stabilizing its position. A tissue volume is represented by its thickness in the direction of the incident light action. Various high power LEDs were used in the tests. The optical part of the transmitting-receiving system is LED diode placed opposite the PIN photodiode PD. Several wavelengths were used for the tests. The results presented herein have been obtained for an ELJ-880-228B emitting light at the wavelength $\lambda=880 \mathrm{~nm}$. This LED was driven with current impulses of parameters as follows: $I_{m}=7 \mathrm{~A}, T_{\mathrm{i}}=1 \mu \mathrm{s}, T_{\text {rep }}=1 \mathrm{~ms}$. The PIN BPW24R photodiode was used as the sensitive photodetector.

The current signal $i_{P D}(t)$ was converted to the corresponding voltage signals and amplified. The voltage $u_{P D}(t)$ appearing during generation of an optical impulse is then subject to analog-to-digital conversion. Reciprocal of the obtained values, marked as $U_{M}$, were collected for particular positions of the scanning system. The relationship occurring between values of $U_{M}$ and levels of $P_{D}$ signal power shows differences in light attenuation connected with absorption and scattering of the transilluminated object. High values of $U_{M}$ prove low levels of $P_{D}$ signal power - in other words, such relation shows strong attenuation for a given position. Figure 11 presents the standardized values of the converted output signal from the photodiode for several $x$ values.

The results obtained as preliminary transillumination images are shown in Fig. 12. The grayness intensity is represented by the output signal values of the fingers examined (F1, F2, F3, F4). The specific isolines illustrate transmission properties of fingers at the used optical radiation. Despite the measuring system simplicity, the imaging obtained was as anticipated. For example, differences between the amplitude variability for fingers without and with joint degenerations were observed.

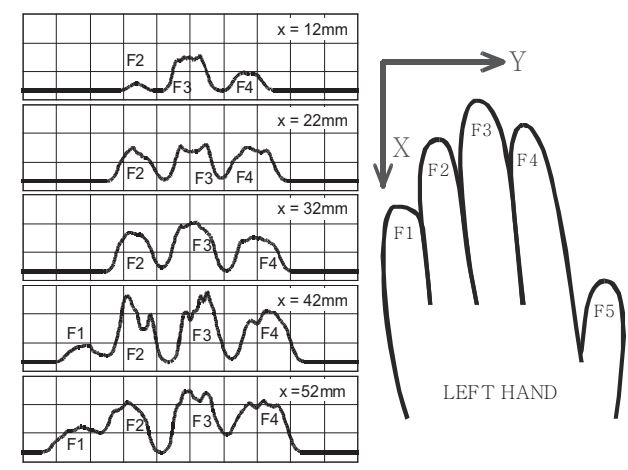

Figure 11: Specification of the output signal dependency on the $y$ location for selected cross-sections with $x$ coordinate.
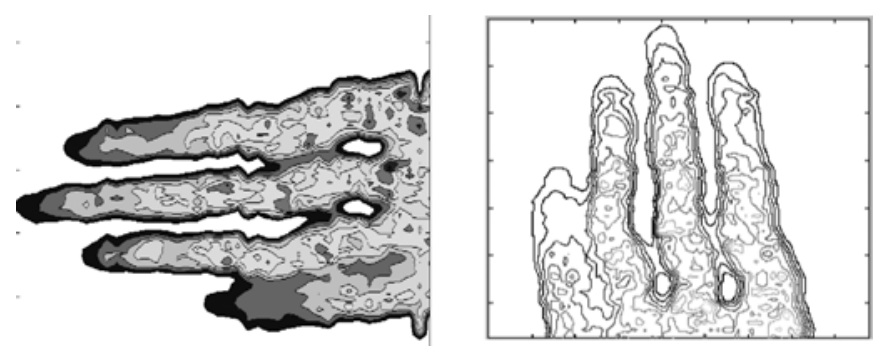

Figure 12: Examples of transillumination images obtained for hand fingers. 
To transilluminate some optically thicker body parts, it is necessary to increase the system's sensitivity, better concentration of the optical bundle and possibility to select the detectable photons. The scanner is being built to achieve this goal.

\section{CONCLUSION}

The practical performance of transillumination is possible due to the transmission supported by forward scattering. However, the backward scattering supports 'illumination of the object from underneath', useful in the diagnostic-therapeutic purposes mentioned above. In cases of peripheral body areas, it is possible to apply transillumination as a simple low-cost method to assist in distinguishing pathological lesions that appeared, for example, in fingers, hands, feet, and teeth. In the transmission variant of the light-object interaction, the objective information on the quantity measured is obtained by means of optoelectronic sensor containing a source of the radiation penetrating the object and receiver of the radiation transmitted through. Numerous issues related to the measurement result interpretation still remain unsolved.

The combined application of various imaging methods is presently developed, allowing for obtaining a more complete set of information on the object, compared to the tests made in one diagnostic technique only. From the combined imaging point of view, the optical imaging may provide information on the functional condition unavailable in X-ray and other techniques. Of course, the progressive development of noninvasive optical imaging and measurements always depends largely on the clinical acceptance of the advanced biomedical technology and engineering. The optical transillumination of biological objects is a diagnostic technique under intensive research. At present, optical transillumination is used in monitoring blood oxygenation, hemorrhage detection, brain imaging, Alzheimer disease diagnostics, mammography, rheumatism and joint inflammable condition monitoring, aiding dental procedures, and improving effects of sclerotherapy. It seems to be reasonable to test transillumination techniques not yet described in any reference literature. The authors of the paper have concentrated their current research on mastering efficient transillumination of thick layers of tissues and building efficient and stable algorithms representing the anatomic and functional properties. The presented preliminary results of finger transillumination tests show that effective transillumination scanning is possible even in a simple system and indicate that the further development of the developed measuring system is appropriate and justified.

\section{REFERENCES}

[1] Enderle, J., Blanchard, S. \& Bronzino, J., Introduction to Biomedical Engineering, Academic Press: San Diego, 2000.

[2] Mannheimer, P.D., The light-tissue interaction of pulse oximetry. Anesthesia \& Analgesia, 105(6), pp. 10-17, 2007. doi: http://dx.doi.org/10.1213/01.ane.0000269522.84942.54

[3] King, R., Optoelectronic sensors in medical applications. Texas Advanced Optoelectronic Solutions (TAOS), Inc, September 2003.

[4] Perez, R.J., Optical sensors (Chapter 8). Design of medical electronic devices, Academic Press: San Diego, pp. 237-73, 2002.

[5] Cysewska-Sobusiak, A., Wiczynski, G., Krawiecki, Z. \& Sowier, A., Role of optical techniques in combined use of selected methods of medical imaging. Opto-Electronics Review, 16(2), pp. 136-146, 2008. doi: http://dx.doi.org/10.2478/s11772-008-0010-7

[6] Duck, F.A., Physical properties of tissue: A comprehensive reference book, Academia Press: San Diego, 1990. 
[7] Cysewska-Sobusiak, A., One-dimensional representation of light-tissue interaction for application in noninvasive oximetry. Optical Engineering, 36, pp. 1225-1233, 1997. doi: http://dx.doi.org/10.1117/1.601242

[8] Cysewska-Sobusiak, A., Powers and limitations of noninvasive measurements implemented in pulse oximetry. Biocybernetics and Biomedical Engineering, 22, pp. 79-96, 2002.

[9] Cysewska-Sobusiak, A., Hulewicz, A., Krawiecki, Z. \& Wiczynski, G., Examples of the application of light-tissue interaction to biomedical engineering. Light in Engineering and the Environment, eds. K. Domke \& C.A. Brebbia, WIT Transactions on the Built Environment, Vol. 121, WIT Press: UK, pp. 223-234, 2011.

[10] Pogue, B.W., McBride, T.O., Osterberg, U.L. \& Paulsen, K.D., Comparison of imaging geometries for diffuse optical tomography of tissue. Optics Express, 4(8), pp. 270-286, 1999.

[11] Yam, F.K. \& Hassan, Z., Innovative advances in LED technology. Microelectronics Journal, 36, pp. 129-137, 2005.

[12] Tirpak, A. \& Fong, A.Y., LED metrology evolves. SPIE Professional, October 2011, pp. 18-21.

[13] Webster, J.G., Design of pulse oximeters, Institute of Physics Publishing: Bristol and Philadelphia, 1997.

[14] Nellcor technology overview: Nellcor Symphony N-3000. The next generation on Nellcor Pulse Oximetry. Nellcor: Pleasanton, 1995.

[15] Prokop, D., Cysewska-Sobusiak, A. \& Hulewicz, A., Application of a multi-sensor set for comparative evaluation of the photoplethysmographic waveforms, Proc. of the First International Conference on Sensor Device Technologies and Applications, Venice-Mestre, Italy, 18-25 July, 2010, IEEE Computer Society, s. 242-245.

[16] Cysewska-Sobusiak, A., Wiczynski, G. \& Jedwabny, T., Specificity of software co-operating with an optoelectronic sensor in a pulse oximeter system. Proc. SPIE, 2634, pp. 172-178, 1995.

[17] USB UI-1240ME datasheet, http://www.ids-imaging.com.

[18] Cysewska-Sobusiak, A. \& Wiczyns ki, G., Preliminary results of using transillumination for optical imaging of hand fingers. Proc. of the Symposium on Photonics Technologies for the 7th Framework Program, Wroclaw, Poland, pp. 333-336, 2006. 